\title{
Synthesis of Photo-degradable Polyphthalaldehyde Macromonomer and Adhesive Property Changes of its Copolymer with Butyl Acrylate on UV-irradiation
}

\author{
Hirokazu Hayashi ${ }^{1 *}$, Hideki Tachi ${ }^{2}$, and Kanji Suyama ${ }^{3^{* *}}$ \\ ${ }^{1}$ Research Division of Applied Material Chemistry, \\ Izumi Center, Osaka Research Institute of Industrial Science and Technology (ORIST), \\ ${ }^{2}$ Research Division of Polymer Functional Materials, \\ Izumi Center, Osaka Research Institute of Industrial Science and Technology (ORIST), \\ 7-1 Ayumino-2, Izumi, Osaka 594-1157, Japan, \\ ${ }^{3}$ Faculty of Liberal Arts and Sciences, Osaka Prefecture University, \\ 1-1 Gakuencho, Nakaku, Sakai, Osaka 599-8531, Japan \\ *hayashi@tri-osaka.jp \\ **suyama@las.osakafu-u.ac.jp
}

\begin{abstract}
We prepared a new polyphthalaldehyde (PPA) macromonomer by introducing a polymerizable methacryloyl group at the terminal of PPA main-chain. Resulting macromonomer was copolymerized with butyl acrylate to obtain pressure-sensitive adhesives (PSAs). We also compared the behavior of the copolymers with those of polymer blends of poly(butyl acrylate) and linear PPA polymers to clarify the role of polymerization. Higher peel strengths were observed for copolymers than those of corresponding polymer blends. As an increase in irradiation time, the strength generally decreased, although once increased for the copolymer films at the early stage. These results suggest that the introduction and the depolymerization of PPA side-chains caused drastic changes in adhesive properties.
\end{abstract}

Keywords: Polyphthalaldehyde, Photo-degradation, Macromonomer, Peel strength, Pressure-sensitive adhesive

\section{Introduction}

Polyphthalaldehydes (PPAs) are known as selfimmolative polymers that can be end-to-end depolymerized (unzipping) [1,2]. Recently, PPAs attract much attention again as a candidate for stimuli-responsible polymers for many applications [3-5].

We have synthesized PPAs with oxime ether terminals, and their photoreactions were investigated [6]. On UV-irradiation both in solution and film state, the depolymerization of the PPAs was confirmed by NMR, UV, and IR spectral measurements. Furthermore, the results of the nanoindentation measurement indicated that the films became elastic on irradiation. Among them, a PPA with 1-acetonaphthone oxime and acetyl terminal (1NaPPA) showed a good depolymerizable behavior.

In this study, we have prepared a new PPA macromonomer 1NaPPAMA by introducing a polymerizable methacryloyl group at the terminal of PPA main-chain. Also, resulting macromonomer was copolymerized with butyl acrylate (BA) to obtain pressure-sensitive adhesives (PSAs) as shown in Scheme 1.

The performance of PSAs such as tack, peel resistance, and share resistance generally depends on the viscoelastic properties caused by the balance of storage and loss moduli [7-11]. These properties are provided by soft and hard molecular structures in the PSAs. The latter enables cohesive interaction between polymer chains in the PSA matrixes [12] and have been often introduced in the form of fillers [13-15], side-chains [16-18], and 

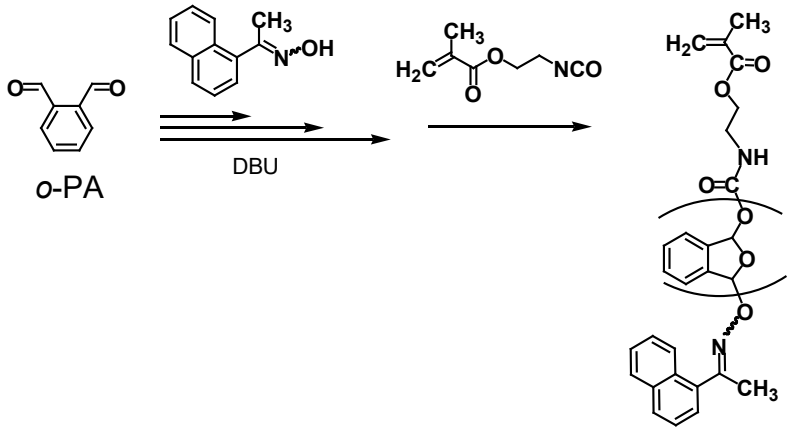

1NaPPAMA
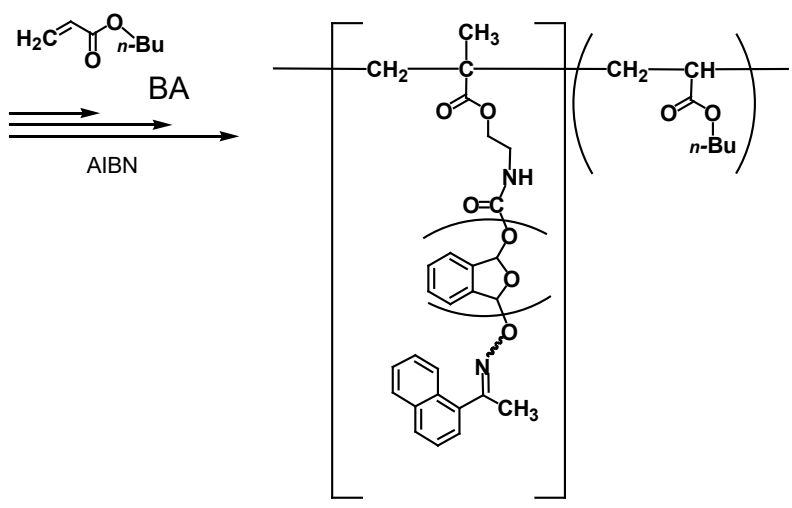

1NaPPAMA-BA copolymers

Scheme 1. Syntheses of 1NaPPAMA and its copolymers with BA.

physically or chemically crosslinking points [19-22].

Because our copolymers 1NaPPAMA-BA involve less flexible and photo-degradable PPA chains, remarkable changes of mechanical property in PSAs are expected. These characteristics are advantageous from the viewpoint of stimulisensitive PSAs [23-28]. Although we have already proposed PSAs composed of oxime-ester based photolabile crosslinkers [29], quite different mechanism and behavior are anticipated. We also compared the behavior of the copolymers with those of polymer blends of PBA and linear PPA polymers such as $1 \mathrm{NaPPA}$ to clarify the role of polymerization.

\section{Experimental}

\subsection{General}

IR and UV spectra were recorded on Jasco FTIR4200, and Shimadzu UV1600PC spectrometers, respectively. NMR spectra were measured by JEOL JNM-ECX400 and Bruker BioSpin Ascend 400 spectrometers.

Number $\left(M_{\mathrm{n}}\right)$ and weight $\left(M_{\mathrm{w}}\right)$ average molecular weights of polymers were obtained by size exclusion chromatography (SEC) with a Tosoh 8020 liquid chromatography system (Tokyo, Japan) composed of two TSKgel GMHXL columns, and a ViscoTech TDA-302 (RI, DP, RALS, LALS) detector with THF eluent and polystyrene standards at $40{ }^{\circ} \mathrm{C}$.

Commercially available reagents were used as received unless otherwise noted. $\mathrm{BA}, o$ dichlorobenzene (DCB), tetrahydrofuran (THF), and $N, N$-dimethylformamide (DMF) were subjected to activated alumina columns before use. 1Acetonaphthone oxime and 1NaPPA were obtained as described previously [6].

\subsection{Preparation of $1 \mathrm{NaPPAMA}$}

In a flask, $4.8 \mathrm{~g}(35.8 \mathrm{mmol})$ of $o$ phthalaldehyde (o-PA) (SP grade for fluorometry, Nacalai, Kyoto, Japan) and $10 \mathrm{~mL}$ of $\mathrm{CH}_{2} \mathrm{Cl}_{2}$ (dehydrated grade, Nacalai) was added under argon, and the flask was cooled to $-80{ }^{\circ} \mathrm{C}$ in an aluminum block cryostat PSL-2500 A (EYELA, Tokyo, Japan). In other vial, $82.4 \mathrm{mg}(0.54 \mathrm{mmol})$ of 1,8 diazabicyclo[5.4.0]undec-7-ene (DBU) and $102 \mathrm{mg}$ $(0.56 \mathrm{mmol})$ of 1-acetonaphthone oxime were dissolved in $3 \mathrm{~mL}$ of $\mathrm{CH}_{2} \mathrm{Cl}_{2}$. Then, $2.5 \mathrm{~mL}$ of the solution was taken out with a syringe and added to the flask dropwise over $20 \mathrm{~s}$, and the mixture was kept stirring at $-80^{\circ} \mathrm{C}$ for $20 \mathrm{~min}$.

The polymerization was terminated by dropping $1 \mathrm{~mL}$ of $\mathrm{CH}_{2} \mathrm{Cl}_{2}$ solution containing $72 \mu \mathrm{L}$ (0.51 mmol) of 2-methacryloyloxyethyl isocyanate (MOI, Showa Denko) over $20 \mathrm{~s}$. After stirring at $-80{ }^{\circ} \mathrm{C}$ for $10 \mathrm{~min}$, the flask was taken out from the cryostat bath and stirred for $20 \mathrm{~min}$ at room temperature. Then, $5 \mathrm{~mL}$ of methanol was added to the flask and removed the solvent under reduced pressure to obtain $6.13 \mathrm{~g}$ of a white solid. The solid was reprecipitated 5 times from methanol after dissolving in $\mathrm{CHCl}_{3}$ to afford $3.69 \mathrm{~g}$ (Conv. $77 \%$ ) of $1 \mathrm{NaPPAMA}$ as colorless powder.

\subsection{Preparation of copolymers of 1 NaPPAMA and BA \\ 1NaPPAMA, BA, and 2,2'- azobisisobutyronitrile were dissolved in a solvent and bubbled with $\mathrm{N}_{2}$ gas. The mixture was heated at $60^{\circ} \mathrm{C}$ until the bubbles raised slowly. Obtained copolymers were reprecipitated 5 times from methanol after dissolving in $\mathrm{CHCl}_{3}$.}


Table 1. Polymerization conditions and characterization of 1NaPPAMA and BA copolymers.

\begin{tabular}{|c|c|c|c|c|c|c|c|c|c|}
\hline \multirow{2}{*}{ Polymer } & \multicolumn{2}{|c|}{ In feed (mol \%) } & \multicolumn{3}{|c|}{ In polymer $(\mathrm{mol} \%)^{\mathrm{a}}$} & \multirow{2}{*}{$\begin{array}{l}\text { R. T. } \\
\text { (h) }\end{array}$} & \multirow{2}{*}{$\begin{array}{l}\text { Conv. } \\
(\%)^{\mathrm{c}}\end{array}$} & \multirow{2}{*}{$\begin{array}{c}M_{\mathrm{n}}^{\mathrm{d}} \\
(\mathrm{kDa})\end{array}$} & \multirow{2}{*}{$\begin{array}{c}M_{\mathrm{w}}^{\mathrm{d}} \\
(\mathrm{kDa})\end{array}$} \\
\hline & 1NaPPAMA & BA & 1NaPPAMA & BA & $o-\mathrm{PA}: \mathrm{BA}$ & & & & \\
\hline $1_{\mathrm{NaPPAMA}}{ }_{0.61-\mathrm{BA}}$ & 0.15 & 99.85 & 0.61 & 99.39 & $0.57: 1$ & 5.5 & 36 & 312 & 559 \\
\hline 1NaPPAMA $_{0.21}$-BA & 0.050 & 99.95 & 0.21 & 99.79 & $0.20: 1$ & 3.7 & 66 & 391 & 1,480 \\
\hline $1_{\mathrm{NaPPAMA}} .05-\mathrm{BA}$ & 0.016 & 99.98 & 0.05 & 99.95 & $0.05: 1$ & 6.0 & 34 & 266 & 781 \\
\hline $\mathrm{PBA}^{\mathrm{e}}$ & 0 & 100 & 0 & 100 & $0: 1$ & 0.8 & 50 & 227 & 787 \\
\hline
\end{tabular}

a) Estimated by ${ }^{1} \mathrm{H}$ NMR area ratio. b) Reaction time at $60{ }^{\circ} \mathrm{C}$ in DCB. c) After reprecipitation. d) From SEC.

e) BA homopolymer.

\subsection{Peel strength measurement}

Polymers were dissolved in toluene (15 wt \%), coated on $50 \mu \mathrm{m}$ PET films with an applicator $(200$ $\mu \mathrm{m}$ gap), and dried overnight in reduced pressure at room temperature. The thickness of the coated films was estimated to be $30 \mu \mathrm{m}$. The films were cut into $10 \mathrm{~mm} \times 80 \mathrm{~mm}$ pieces, overlapped with another $2 \mathrm{~mm}$ PET plate, and pressed by a roller with $2 \mathrm{kgf}$. After leaving for $20 \mathrm{~min}$, the films were irradiated with a Hamamatsu Photonics LC5 $\mathrm{Hg}$-Xe lamp (Shizuoka, Japan) through of the PET plate. The light intensity was measured by an Orc UV-M03 illuminometer (Tokyo, Japan) and found to be $150 \mathrm{~mW} / \mathrm{cm}^{2}$ at $365 \mathrm{~nm}$. $180^{\circ}$ Peel strength was evaluated using an Instron 5582 Materials Testing System with $100 \mathrm{~N}$ loadcell at $300 \mathrm{~mm} / \mathrm{min}$. For all samples, 3 runs were carried out.

\section{Results and discussion}

3.1. Synthesis and characteristics of macromonomer 1NaPPAMA

1NaPPAMA was prepared by anionic polymerization of $o$-PA using 1-acetonaphthone oxime as initiator and DBU as a catalyst as described in our previous study [6], except the terminating reagent. In the present study, the polymerization was terminated with MOI which has both isocyanato and methacryloyl groups. Purified polymer was obtained by repeated reprecipitation.

In ${ }^{1} \mathrm{H}$ NMR spectrum of resulting polymer, small peak at $2.4 \mathrm{ppm}$ assignable to $\mathrm{CH}_{3}$ unit in oxime moiety is observed along with broad bands (6.4-7.6 ppm) due to aromatic and $\mathrm{CH}$ groups in main-chains. Also, small peaks at 1.9, 4.3, 5.6, and $6.1 \mathrm{ppm}$ due to $\mathrm{CH}_{3}, \mathrm{CH}_{2}$, and $\mathrm{CH}_{2}=\mathrm{C}$ groups in MOI moiety appeared. In addition, the UV spectrum of obtained polymer solution has a shoulder around $290 \mathrm{~nm}$ due to naphthyl unit. These results clearly show the presence of both naphthyl and methacryloyl terminals.

From SEC, $M_{\mathrm{n}}$ and $M_{\mathrm{w}}$ values were 12.8 and $23.5 \mathrm{kDa}$, respectively. From $M_{\mathrm{n}}$ value, the repeating units of $o$-PA in 1NaPPAMA are estimated to be 93 .

\subsection{Preparation of 1 NaPPAMA and BA copolymers}

In order to obtain polymers for PSAs, 1NaPPAMA was copolymerized with BA in freeradical mode. In preliminary experiments, we used THF, DMF, and DCB as a solvent. Because DCB afforded the highest yield, we used DCB as a polymerization solvent in further experiments.

The polymerization condition and characteristics of the copolymer with different ratio of 1NaPPAMA and BA are summarized in Table 1. After purification, ${ }^{1} \mathrm{H}$ NMR spectra showed that both 1NaPPAMA and BA units were successfully incorporated in the copolymers. Based on that $1 \mathrm{NaPPAMA}$ contains 93 units of $o$-PA, the ratios of $o$-PA to BA in copolymers are calculated as shown in Table 1. It is estimated that one polymer chain of $1_{\mathrm{NaPPAMA}} \mathrm{N}_{0.61}-\mathrm{BA}, \mathrm{NaPPAMA}_{0.21}$-BA, and $1 \mathrm{NaPPAMA}_{0.05}$-BA have $15,6.4$, and 1.0 PPA sidechains, respectively.

3.3. Comparison of copolymers and polymer blends in peel strength

Films of 1NaPPAMA-BA copolymers were prepared for peel strength measurement. Although films of $1 \mathrm{NaPPAMA}_{0.05}$-BA were clear, $1 \mathrm{NaPPAMA}_{0.21} \mathrm{BA}$ and $1 \mathrm{NaPPAMA}_{0.61} \mathrm{BA}$ gave cloudy and opaque films, respectively.

Peel strengths of 1NaPPAMA-BA copolymers are plotted as a function of irradiation time in Fig. 1. Before irradiation, both $1 \mathrm{NaPPAMA}_{0.21}-\mathrm{BA}$ and $1_{\text {NaPPAMA }}$.05 -BA films showed much higher peel strength compared to PBA, probably due to PPA side-chains which aggregate to form hard segments. In case of $1 \mathrm{NaPPAMA}_{0.61}-\mathrm{BA}$, the initial peel 


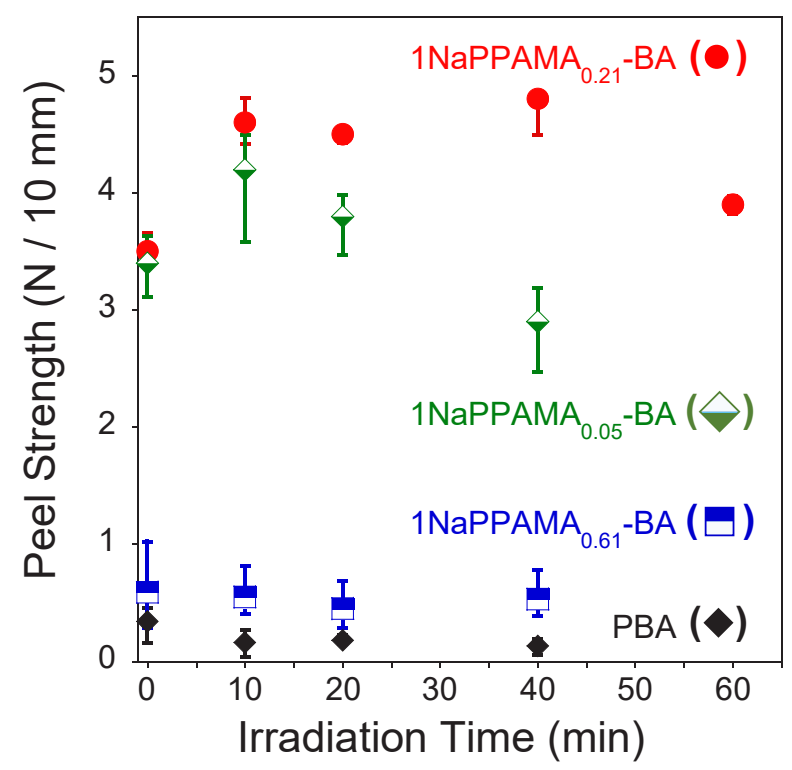

Fig. 1. Peel strength changes of 1NaPPAMA-BA copolymer films on irradiation. Symbols in error bars indicate the averaged values of 3 runs.

strength was $0.6 \mathrm{~N}$ as low as PBA, suggesting that too much PPA fraction caused the copolymer harder as a PSA.

On irradiation of $1 \mathrm{NaPPAMA}_{0.21}$-BA, peel strengths increased from 3.5 to $4.5 \mathrm{~N}$, and the strength decreased on irradiation for $60 \mathrm{~min}$. Similar trend is observed for $1 \mathrm{NaPPAMA}_{0.05}-\mathrm{BA}$, where the peel strength once increased on $10 \mathrm{~min}$ irradiation and then decreased. Considering that the photo-induced depolymerization of 1NaPPA films proceeded on irradiation for $15 \mathrm{~min}$ or above [6], it is expected that photo-degradation of PPA chains in copolymers also proceeds with similar irradiation time. Thus, the increase in peel strength for both copolymers will correspond to the depolymerization of PPA side-chains, although the reason is unknown at present.

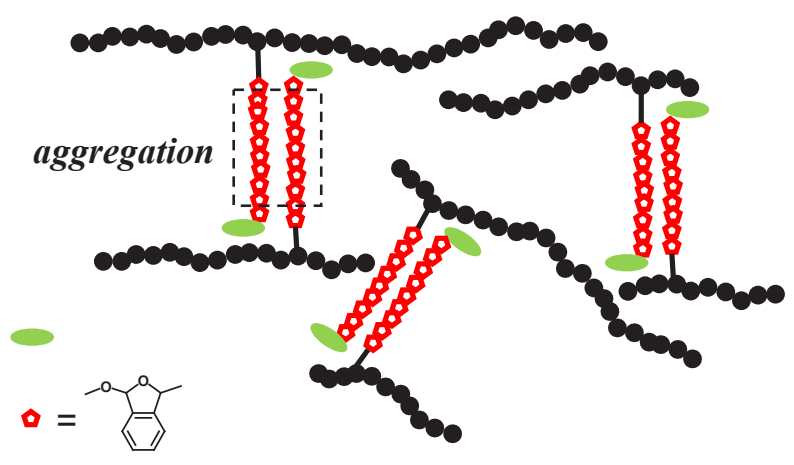



Fig. 3. Peel strength changes of polymer blend films on irradiation. BA unit : $o$-PA unit $=1: 0.2(\mathrm{~mol} / \mathrm{mol})$. Symbols in error bars indicate the averaged values of 3 runs.

Longer time irradiation caused the decrease in peel strength for both copolymers, which would be due to the disappearance of hard PPA segments as shown in Fig. 2. The degradation of BA mainchains might contribute the decrease, because the slight decrease is often observed for PBA in the previous [29] and present study.

Films of $1 \mathrm{NaPPAMA}_{0.61}$-BA did not show the increase in the peel strength. This result suggests that too much PPA side-chains in $1 \mathrm{NaPPAMA}_{0.61^{-}}$ BA made the matrix glassy to detect the changes of peel strength.

In order to clarify the effect of covalent bonding of PPA chains and BA chains, we investigated the peel strengths of PSAs composed of PBA/1NaPPA and PBA/1NaPPAMA polymer blends with the same ratio of BA to $o$-PA units as

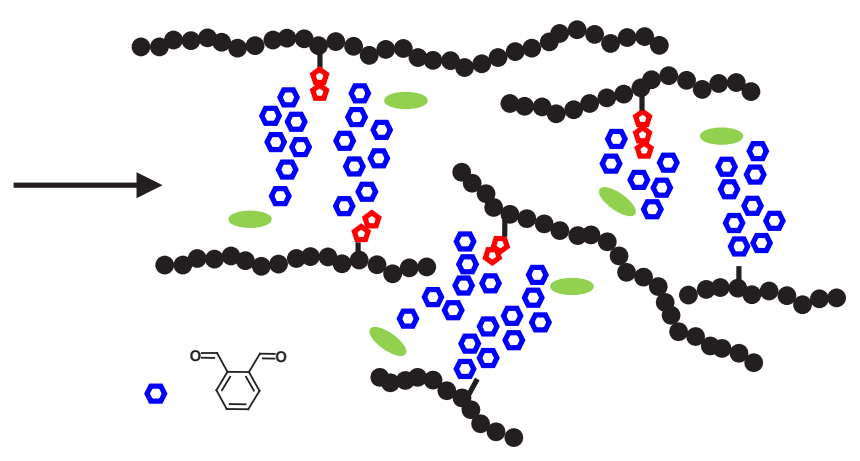

Fig. 2. Schematic illustration of photo-degradation of 1NaPPAMA-BA copolymers. 

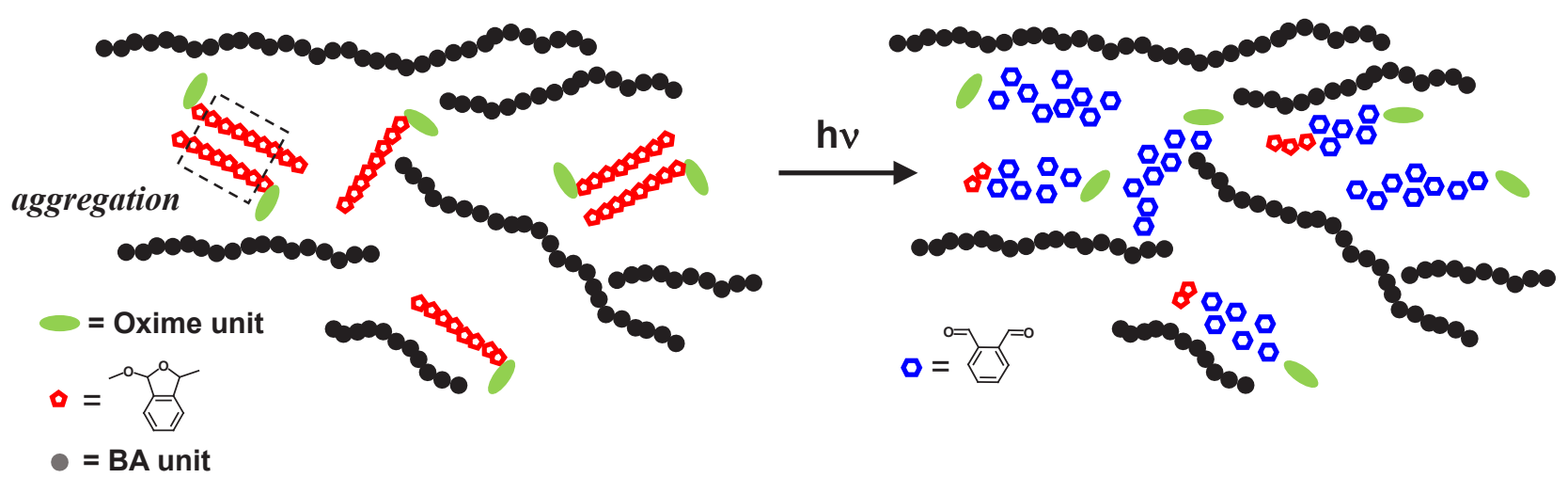

Fig. 4. Schematic illustration of photo-degradation of polymer blends.

that in $1 \mathrm{NaPPAMA}_{0.21}$-BA. Figure 3 shows the changes of peel strength of the polymer blends. The initial peel strengths of $\mathrm{PBA} / o-\mathrm{PA}$, PBA/1NaPPA, and PBA/1NaPPAMA were 0.8, 2.1, and $2.6 \mathrm{~N}$, respectively, located between those of $1 \mathrm{NaPPAMA}_{0.21}$-BA and PBA. It was considered that these results were caused by the increase of hard segment in PSA matrixes.

On irradiation, the peel strengths for both PSAs gradually decreased with an increase in irradiation time and got closer to the value of PBA/ $/$-PA, $0.8 \mathrm{~N}$. This trend was different from those of $1_{\text {NaPPAMA }}{ }_{0.21}$-BA and $1_{\text {NaPPAMA }}$. $_{05}$-BA films as illustrated in Fig. 4.

Failure modes of the PSAs are consistent with this assumption. For copolymers $1 \mathrm{NaPPAMA}_{0.21^{-}}$ BA, interfacial mode was preferential, although $1 \mathrm{NaPPAMA}_{0.05}$-BA and blended polymers detached with cohesive mode. These behaviors did not change before and after irradiation for all coated films.

In conclusion, we have prepared a macromonomer 1NaPPAMA and its copolymers with BA, and investigated the peel strength changes on irradiation to evaluate their pressure-sensitive adhesive properties. Higher peel strengths were observed for copolymers than those of corresponding polymer blends. As an increase in irradiation time, the strength generally decreased, although once increased for $1 \mathrm{NaPPAMA}_{0.21}$-BA and $1 \mathrm{NaPPAMA}_{0.05}$-BA copolymer films at the early stage. These results suggest that the introduction and the depolymerization of PPA side-chains caused drastic changes in adhesive properties.

\section{Acknowledgements}

This work was supported by Ogasawara
Foundation. The authors also thank to Dr. Akiko Nakahashi of ORIST for SEC analysis.

\section{References}

1. H. Ito and C. G. Willson, Polym. Eng. Sci., 23 (1983) 1012.

2. F. Wang and C. E. Diesendruck, Macromol. Rapid Commun., 39 (2018) 1700519.

3. R. E. Yardley, A. R. Kenaree, and E. R. Gillies, Macromolecules, 52 (2019) 6342.

4. J. P. Lutz, O. Davydovich, M. D. Hannigan, J. S. Moore, P. M. Zimmerman, and A. J. McNeil, J. Am. Chem. Soc., 141 (2019) 14544.

5. V. Eriksson, M. A. Trojer, S. Vavra. M. Hulander, and L. Nordstierna, J. Colloid Interface Sci., 579 (2020) 645.

6. H. Hayashi, H. Tachi, and K. Suyama, J. Photopolym. Sci. Technol., 33 (2020) 269.

7. E. P. Chang, J. Adhes., 34 (1991) 189.

8. I. Benedek, "Development and Manufacture of Pressure-Sensitive Products", Marcel Dekker, New York (1998).

9. I. Benedek and M. M. Feldstein Ed., "Fundamentals of Pressure Sensitivity", CRC Press, Boca Raton, Florida (2008).

10. M. M. Feldstein, E. E. Dormidontova, and A. R. Khokhlov, Prog. Polym. Sci., 42 (2015) 79.

11. S. Mapari, S. Mestry, and S. T. Mhaske, Polym. Bull., (2020) DOI: 10.1007/s00289-020-033051.

12. R. Li, J. A. T. Loontjens, and Z. Shan, Eur. Polym. J., 112 (2019) 423.

13. V. G.-Pacios, Y. Iwata, M. Colera, and J. M. M.Martínez, Int. J. Adhes. Adhes., 31 (2011) 787.

14. M. K. Shamsabadi and M. R. Moghbeli, Int. J. Adhes. Adhes., 78 (2017) 155.

15. Z. Dastjerdi, E. D. Cranston, and M. A. Dubé, 
Int. J. Adhes. Adhes., 81 (2018) 36.

16. S. Yamaguchi, S. Tabuchi, S. Kawahara, and H. Murakami, Chem. Lett., 45 (2016) 463.

17. S. Yamaguchi, R. Nakanishi, M. Nanchi, S. Kawahara, and H. Murakami, Chem. Lett., 47 (2018) 344.

18. Y. Wang, F. Weng, J. Li, L. Lai, W. Yu, S. J. Severtson, and W.-J. Wang, ACS Omega, 3 (2018) 6945.

19. C. Fang and Z. Lin, Int. J. Adhes. Adhes., 61 (2015) 1.

20. C. Fang, Y. Jing, Y. Zong, and Z. Lin, J. Adhes. Sci. Technol., 31 (2017) 858.

21. Y. Wang, K. Jia, C. Xiang, J. Yang, X. Yao, and Z. Suo, ACS Appl. Mater. Interfaces, 11 (2019) 40749.

22. E. S. Kim, D. B. Song, K. H. Choi, J. H. Lee, D. H. Suh, and W. J. Choi, J. Polym. Sci., 58 (2020)
3358.

23. R. Vendamme, N. Schüwer, and W. Eevers, $J$. Appl. Polym. Sci., 131 (2014) 40669.

24. Q. Chen, Q. Yang, P. Gao, B. Chi, J. Nie, and Y. He, Ind. Eng. Chem. Res., 58 (2019) 2970.

25. P. Hao, T. Zhao, L. Wang, S. Liu, E. Tang, and X. Xu, Prog. Org. Coat., 137 (2019) 105281.

26. M. Koike, M. Aizawa, N. Akamatsu, A. Shishido, Y. Matsuzawa, and T. Yamamoto, Bull. Chem. Soc. Jpn., 93 (2020) 1588.

27. G.-S. Shim, J.-S. Kim, J.-H. Back, S.-W. Jang, J.-W. Park, H.-J. Kim, J.-S. Choi, and J.-S. Yeom, Int. J. Adhes. Adhes., 96 (2020) 102445.

28. P. Bednarczyk, K. Mozelewska, and Z. Czech, Int. J. Adhes. Adhes., 102 (2020) 102652.

29. K. Suyama and H. Tachi, J. Photopolym. Sci. Technol., 28 (2015) 45. 